Möbius calculates the values of the successive ranges formed by these centres, and finds that

$\left(D M M^{\prime} M^{\prime \prime}\right)=5,\left(M^{\prime} M^{\prime \prime} M^{\prime \prime \prime}\right)=\left(M^{\prime} M^{\prime \prime} M^{\prime \prime \prime} M^{\prime \prime \prime \prime}\right)=$ etc. $=\frac{25}{21}$.

The last two chapters of the Calculus are devoted to a discussion of the principle of duality. This principle, which is now so well known, was only being investigated when Möbius wrote in 1827 . As a consequence of the theory of reciprocal polars, it was first stated by Poncelet in the year 1818, and as an independent principle by Gergonne in 1826, and the Barycentric Calculus was published in 1827. In his preface Möbius states that before the part of his work dealing with duality was sent to press he had heard of the papers of Poncelet and Gergonne, but had not seen them. It is unnecessary to give an account of his treatment of the subject; but it may be well to state that he enunciates the principle quite generally as a characteristic property of space and treats the subject independently of the properties of conic sections.

Second Meeting, December 11, 1891.

Professor J. E. A. Steggald, M.A., President, in the Chair.

Note on an approximate fractional expression for the expansion of $(1+x)^{n}$ to any odd number of terms.

By Professor J. E. A. Steggall.

In Bonnycastle's Arithmetic a rule is given for finding any root of a number by approximation which in substance reduces to this statement:- If $a$ and $b$ are nearly equal, then the $n^{\text {th }}$ root of $a / b$ is nearly equal to

$$
\frac{(n+1) a+(n-1) b}{(n+1) b+(n-1) a}
$$

or, if we call $a / b, 1+x$, we have $(1+x)^{\frac{1}{n}}=\frac{2 n+(n+1) x}{2 n+(n-1) x}$

For example, let $a=2, b=1, n=3$, then an approximation to the cube root of 2 is

$$
\frac{4.2+2.1}{4.1+2.2}=\frac{5}{4}
$$


a first approximation which can he used as a basis for a second thus :-

$$
2^{\frac{1}{3}}=\frac{5}{4}\left(\frac{128}{125}\right)^{\frac{1}{3}}
$$

and applying the rule

$$
\begin{aligned}
2^{\frac{1}{3}} & =\frac{5}{4} \cdot \frac{4128+2.125}{4.125+2.128}, \text { nearly, } \\
& =\frac{635}{504} \text { nearly. }
\end{aligned}
$$

This result is obtained with little labour, and is correct to six decimal places.

It is clear that we may test the accuracy of the approximation by expansion, and we accordingly find that the difference in the two sides of (1) is

$$
\frac{x^{3}\left(n^{2}-1\right)}{12 n^{3}}
$$

an error in the given example of about

$$
\frac{3^{3} \cdot 8.5}{125^{3} .12 .3^{3} .4} \text { or } \cdot 0000001 \text {. }
$$

A general problem is at once presented, namely, to find a fraction

$$
\frac{1+a_{1} x+a_{2} x^{2}+\ldots+a_{r} x^{r}}{1+b_{1} x+b_{2} x^{2}+\ldots+b_{r} x^{r}}
$$

which on expansion shall give the value of $(1+x)^{n}$ correct to $(2 r+1)$ terms. Of course for a direct solution we have $2 r$ linear equations to determine the $a^{\prime} s$ and $b^{\prime} s$, and these equations $I$ succeeded in solving. At the time, I had no opportunity of consulting Gauss, but since the paper was read I find that my result is included in one of his on hypergeometrical series, and is an exact theorem : that is to say, the numerator and denominator of the fraction (2) are the first $r+1$ terms of two hypergeometrical series of which the next $r$ terms are zero, and the finite terms then recommence and continue ad infinitum. This of course explains the accordance to $2 r+1$ terms when the division of and by the first $r+1$ terms is effected.

The final result is

$$
(1+x)^{n}=\frac{\frac{1+(n+r) r}{2 r} x+\frac{(n+r)(n+r-1) r(r-1)}{2 r(2 r-1) 1} x^{2}+\ldots \text { ad infinitum }}{1-\frac{(n-r) r}{2 r} x+\frac{(n-r)(n-r+1) r(r-1)}{2 r(2 r-1) 1.2} x^{2}+\ldots \text { ad infinitum }}
$$

where the factor $(r-r)$ in the $(r+2)^{t h}$ terms causes evanescence until the introduction of $(2 r-2 r)$ in the denominator of the $(2 r+2)^{2 h}$ terms produces a finite, thereafter, result. 
It may be interesting to write down a few cases for the example already considered, namely $(1+x)^{1 / 3}$;

the first result is

the second

$$
\begin{gathered}
\frac{3+2 x}{3+x} \\
\frac{1+7 x / 6+7 x^{2} / 27}{1+5 x / 6+5 x^{2} / 54} \\
\frac{1+5 x / 3+7 x^{3} / 9+7 x^{3} / 81}{1+4 x / 3+2 x^{2} / 9+2 x^{3} / 81} .
\end{gathered}
$$

the third

In Gauss's notation the identity is

$$
(1+x)^{n}=\frac{\mathrm{F}(-n-r,-r,-2 r,-x)}{\mathrm{F}(n-r,-r,-2 r,-x)},
$$

of which a more general case will be found in his Methodus nova integralium valores per approximationem inveniendi, $\$ 40-42$.

\section{On the history and degree of certain geometrical approximations.}

\section{PART I.}

\section{By A. J. Pressland, M.A.}

According to Luther's translation 1 Kings vi. 31 should read"At the entrance of the choir he made two doors with pentagonal door-posts." This is probably a wrong translation, for nowhere on Asiatic monuments of this time has a pentagon been found. Prof. A. Merx in Cantor's Vorlesungen über Geschichte der Mathematik, Vol. i., p. 91 .

It is probable that the construction of the regular pentagon is due to the Pythagorean school as a consequence of I. 47. (Ibid, p. 151.)

Various approximations to the arc of a regular pentagon in a circle have been given. Thus (fig. 8) a diameter $\mathrm{AB}$ of a circle is divided into fre equal parts at $E, D$, etc. $O n A B$ an equilateral triangle is erected giving the vertex $C$. $C D$ is drawn to cut the circumference in $P$, and $A P$ is assumed to be the arc of the regular inscribed pentagon. By dividing $\mathrm{AB}$ into $n$ equal parts and joining $C$ to the second of these from $A$, an approximation to the arc of the regular $n$-gon is obtained.

The first mention of this construction is in Les fortifications of Chevalier Antoine de Ville (achevé d'imprimer, 1er Août, 1628). In this, however, the point $C$ is joined to the point $E$ and the resulting arc doubled. On page 29 the following occurs: "Ce probleme ne 